\title{
Reaching new heights: Comparing interpretation bias modification to exposure therapy for extreme height fear
}

\author{
Shari A. Steinman and Bethany A. Teachman \\ University of Virginia
}

\section{Abstract}

Objective-Cognitive models of anxiety disorders posit that biases in interpretation maintain, and potentially cause, anxiety. This study tested whether it is possible to decrease height fear symptoms through cognitive bias modification for interpretations (CBM-I). Additionally, the clinical utility of CBM-I was tested by comparing it to an already established treatment: exposure therapy.

Method-Extremely height fearful $(N=110)$ individuals participated in the study. Acrophobic symptoms were measured before and after two sessions of CBM-I, and compared to the standard treatment for acrophobia (exposure therapy), a combination of CBM-I and exposure therapy, and a Control condition.

Results-In line with hypotheses, participants in the three active conditions showed greater response to treatment than the Control condition in height-relevant interpretation bias, symptoms, and behavioral avoidance on a height stressor, with few differences between the active conditions. Further, symptom change was mediated by change in interpretation bias.

Conclusions-Overall, findings suggest that different pathways to fear reduction (exposure vs. shifting interpretations) can lead to similar reductions in height fear. This study provides the first evidence that directly shifting cognitive processing, even with no therapist involvement, can reduce symptoms as effectively as the gold standard, therapist-directed exposure therapy.

\section{Keywords}

cognitive bias modification; information processing; height fear; exposure; interpretation; acrophobia

Exposure therapy, in which people confront their feared stimuli and try to remain in the situation until their fear declines, is currently the gold standard for phobia treatment. Metaanalyses and literature reviews have shown that the beneficial effects of exposure are robust and gains are maintained or improved over time (e.g., Choy, Fyer, \& Lipsitz, 2007;

Wolitzky-Taylor, Horowitz, Powers, \& Telch, 2008). Despite the well-documented success of exposure therapy, many phobic individuals do not improve, or find confronting their feared stimuli aversive, and consequently drop out of or avoid treatment (e.g., Choy et al.). In fact, data from the Epidemiologic Catchment Area study showed that approximately 70\% of people with diagnosable phobias had not sought treatment, and of those who had, less 
than 50\% sought services specific to psychological health (see Wolitzky-Taylor et al. for review). Given these limitations, there is a clear need to explore alternate approaches to phobia treatment.

The current study evaluates a new approach to the treatment of phobias: cognitive bias modification for interpretations (CBM-I) for individuals with extreme height fear. To examine the utility of modifying interpretation biases, height fear and related symptoms were measured before and after two sessions of CBM-I, and compared to an Exposure Only condition, a combination of CBM-I and exposure therapy (labeled CBM-I+Exposure), and a Control condition.

\section{Acrophobia and interpretation bias}

Acrophobic individuals have biases in their judgment such that they tend to overestimate danger and doubt their ability to cope in height-relevant situations. For example, they report higher estimates of the likelihood of falling from heights and higher estimates of the probability of injury resulting from heights compared to non-phobic control participants (Menzies \& Clark, 1995; Williams \& Watson, 1985). Additionally, height-fearful individuals tend to interpret ambiguous situations related to heights in a negative way, and this negative interpretation bias has been shown to predict fear and avoidance on actual heights (Steinman \& Teachman, 2011). The current study expands on this correlational research by attempting to directly modify height fear-relevant threat interpretations and examining the effects on subsequent height fear. Note that we define interpretive bias as cognition that resolves disorder-relevant ambiguity (e.g., ambiguity tied to one's emotional response, safety, ability to cope, or personal vulnerability). The experimental intervention in this study attempts to modify interpretations of ambiguous scenarios concerning whether or not heights are dangerous, and whether or not individuals can cope with their anxiety while on heights. Modifying interpretations is expected to be an effective tool for reducing height fear given the centrality of biased interpretations in cognitive models of anxiety (e.g., Beck, Emery, \& Greenberg, 1985). As Beck and Clark (1997) state, "it is the propensity of this information processing apparatus to inappropriately generate threat meaning assignments to innocuous stimuli that is the main problem that must be rectified in the treatment of anxiety disorders" (p. 51).

A growing number of studies have shown that it is possible to directly manipulate interpretive biases using brief computerized paradigms in which participants read and imagine themselves in a string of emotionally ambiguous scenarios that are resolved in a non-threatening way (a paradigm referred to in the current study as Scenario Training). For instance, researchers have used modifications of Scenario Training to induce healthier interpretations in participants with high levels of social anxiety (Murphy, Hirsch, Mathews, Smith, \& Clark, 2007), trait anxiety (Mathews, Ridgeway, Cook, \& Yiend, 2007), spider fear (Teachman \& Addison, 2008), anxiety sensitivity (Steinman \& Teachman, 2010), obsessive compulsive symptoms (Clerkin \& Teachman, 2011), and worry (Hirsch, Hayes, \& Mathews, 2009). Moreover, there is mounting evidence that Scenario Training can reduce subsequent reports of anxiety symptoms on questionnaires (e.g., among individuals with high levels of worry: Hirsch et al.; trait anxiety: Mathews et al.; anxiety sensitivity: 
Steinman \& Teachman). Complementing the effects of Scenario Training, Beard and Amir (2008) created an additional paradigm (Interpretation Modification Paradigm, or IMP) to train participants high in social anxiety symptoms to associate ambiguous sentences with words indicating a benign interpretation. Because using multiple CBM-I approaches may strengthen effects by targeting multiple facets of interpretation bias (see Hirsch et al., 2009), a combination of Scenario Training and IMP was used in the current study. Additionally, to our knowledge, CBM-I has only been compared to Control or No Training conditions, and other computerized treatments (e.g., Bowler et al., 2012). A logical next step is to compare CBM-I to an already established, gold standard treatment.

Despite the theoretical connection between modifying interpretations and traditional cognitive models of anxiety disorders (e.g., Beck \& Clark, 1997), attempting to reduce anxiety through direct modification of interpretation biases is a considerable departure from more conventional anxiety reduction approaches. Specifically, it has been theorized that inducing negative affect is necessary for the treatment of anxiety disorders (e.g., Foa \& Kozak, 1986). CBM-I is quite different because state anxiety often does not change from pre- to post-training (e.g., Mathews et al., 2007; Steinman \& Teachman, 2009; Teachman \& Addison, 2008). Further, it has been posited that "verbal mediation," usually achieved through traditional cognitive restructuring that involves reflecting upon and discussing one's personal cognitions, is necessary for reducing anxiety (e.g., Beck \& Clark). However, interpretation bias modification is thought to alter threat-meaning assignment (e.g., interpretations) in a non-traditional way - through repeated practice making positive interpretations (and rejecting negative interpretations) of ambiguous situations presented on a computer, without the assistance of a therapist or explicitly re-evaluating one's personal, threat-oriented interpretations. This points to alternate avenues to fear reduction. Notably, it has been suggested that specific fears are less cognitive in nature (e.g., involve less rumination and worry) relative to other types of anxiety, and as such, may be less amenable to CBM (see discussion in Reese, McNally, Najmi, \& Amir, 2010). By testing the effect of CBM-I on height fear, the current study evaluates the importance of cognition in maintaining specific fear.

\section{Overview and Hypotheses}

Individuals with extreme height fear were assigned to two sessions of CBM-I Only, Exposure Only, CBM-I+Exposure, or a Control condition. It was expected that following treatment, participants in the three active treatment conditions (but not the Control condition) would show significant reductions in interpretation biases, height fear and related symptoms. Additionally, it was hypothesized that the CBM-I+Exposure condition would show the greatest reductions in all outcome measures. MacLeod, Koster, and Fox (2009) predict that using cognitive bias modification, such as CBM-I, with traditional treatments will result in "therapeutic synergies" in which conventional therapy approaches and bias modification positively impact each other. Given that threat meaning assignment is thought to play a central role in anxiety disorders (e.g., Beck \& Clark, 1997), and given the wide support for exposure procedures in the treatment of anxiety (e.g., Wolitzky-Taylor et al., 2008), a treatment that both targets the ascription of threat meanings (through CBM-I) and includes exposures may outperform a treatment with only one of these two components. 
Note that we expected both CBM-I and exposure to change interpretation bias, given that cognitive-behavioral treatments are theorized to change cognition (e.g., Beck \& Clark, 1997). In fact, in a review of the literature, Hofmann (2008) stated that "exposure therapy is mediated through changes in cognitions" (p. 205). Finally, note that in the CBM-I+Exposure condition, participants completed each individual intervention for half as much time as participants in either of the stand-alone intervention conditions. Thus, we are not able to fully separate the effects of combining the interventions from that of time spent in each intervention. This design choice was made because of the importance of keeping total treatment time equivalent across conditions, which we felt was ultimately more important than keeping each intervention component's dose equivalent.

\section{Methods}

\section{Participants and recruitment}

Extremely height fearful students and community members were recruited through the university's psychology department participant pool, flyers, and newspaper advertisements. Individuals were offered course credit or $\$ 50$ compensation for participation. Potential participants completed the Acrophobia Questionnaire-Anxiety subscale (AQ-Anxiety; Cohen, 1977). Individuals had to score at least a 45.45 on the AQ-Anxiety (i.e., within one standard deviation below the mean in a previous acrophobic sample; Cohen, 1972) to be considered for study inclusion ${ }^{1}$. Individuals who met criteria were screened over the phone using the Specific Phobia section of the Structured Clinical Interview for the Diagnostic and Statistical Manual of Mental Disorders (4th ed., SCID; First, Spitzer, Gibbon, \& Williams, $1994)^{2}$. Participants included in the study scored in the subthreshold or threshold range for all criteria on the SCID during the phone screen. However, ten individuals participated in the study despite not endorsing one criterion, so to be conservative, we describe our sample as "extremely height fearful" rather than "subthreshold to threshold acrophobic," despite our sample being similar to a diagnosed sample. In fact, our sample's mean on the AQ-Anxiety at baseline $(M=62.30 ; S D=12.73)$ was slightly above that of a previous, diagnosed acrophobic sample $(M=61.30 ; S D=15.85$; Cohen, 1972).

See Figure 1 for a Consolidated Standards of Reporting Trials (CONSORT) diagram that details exclusion and attrition during recruitment. The final sample included 110 participants (75.5\% female). Eighty participants were recruited through the psychology department participant pool, and 30 were recruited through flyers or newspaper advertisements. The mean age of the sample was 23.63 years $(S D=11.38$, range $=18-67)$. The reported race of the participants was White (68.2\%), Asian (18.2\%), Black/African American (7.3\%), multiple ethnicities $(4.5 \%)$ and other (1.8\%). This study received approval from, and complies with, the university's Institutional Review Board (IRB).

\footnotetext{
${ }^{1}$ To reduce the number of participants that were screened over the phone and unlikely to meet inclusion criteria, potential participants completed three modified questions from the SCID at the same time that they completed the AQ-Anxiety, asking individuals to rate whether: 1) they are more afraid of heights than they should be or than makes sense, 2) they go out of their way to avoid heights, and 3) their feelings towards heights distress them or get in the way of things they want to do. The items used a 0 (not at all) to 6 (extremely) scale, and participants had to endorse at least a "2" on one or more of the three questions to be phone screened.

${ }^{2}$ The first author and trained research assistants administered the Specific Phobia Section of the SCID. The research assistants did many practice phone screenings with the first author and with each other to enhance inter-rater consistency. Further, questions about phone screens were routinely reviewed with the first author, though inter-rater reliability was not formally established.
} 


\section{Materials ${ }^{3}$}

Unless otherwise noted, all measures listed below were administered during a baseline assessment, and again at a post-intervention assessment.

\section{Height fear and state fear measures}

The Acrophobia Questionnaire (AQ; Cohen, 1977) is a 40-item Likert-type questionnaire that asks participants to rate their anxiety (AQ-Anxiety) and avoidance (AQ-Avoidance) associated with 20 height-relevant situations (e.g., "riding a Ferris wheel"). The full AQ has good psychometric properties and is widely used (Baker, Cohen, \& Saunders, 1973; Bourque \& Ladouceur, 1980). Across the preselection, baseline, post-intervention, and follow-up assessments, Cronbach's alpha for the AQ-Anxiety and AQ-Avoidance subscales ranged from .72-.92 (average $=.81)$.

The Attitudes Towards Heights Questionnaire (ATHQ; originally Abelson \& Curtis, 1989, with minor modifications to the wording reported in Coehlo, Santos, Silvério, \& Silva, 2006) is a 6-item measure in which individuals read pairs of dichotomous adjectives describing ways people may feel about heights (e.g., "Good/Bad," "Safe/Dangerous"), and rate how they feel about elevated places on a scale of 0 (which corresponds with the first adjective) to 10 (which corresponds with the second adjective). The ATHQ has been used in several height fear treatment studies and is sensitive to treatment effects (Coehlo et al., 2006; Emmelkamp, Bruynzeel, Drost, \& van der Mast, 2001). In the current study, Cronbach's alpha for the ATHQ was .86 at the baseline assessment, and .91 at the postintervention assessment.

The Fear Subscale of the Positive and Negative Affect Schedule-Expanded Form (PANASFS; Watson \& Clark, 1994) is a 6-item self-report measure of state fear based on adjective ratings. The PANAS has good reliability and validity (Waston \& Clark). In the current study, the PANAS-FS was used to examine change in affect following the height stressor. Across administrations in the baseline and post-intervention assessments, Cronbach's alpha ranged from .60-.93 (average $=.79$ ). Note that we used the PANAS-FS as opposed to the frequently used Subjective Units of Distress Scale (SUDS) to obtain a more psychometrically sound measure of subjective fear (given that the PANAS-FS includes more than one item, likely providing a more reliable estimate of distress, and given that it has established strong psychometric properties). Additionally, using the PANAS-FS matches how fear was assessed during the stair-climbing BAT in Steinman and Teachman (2011).

\section{Interpretation bias measures}

The Heights Interpretation Questionnaire (HIQ; Steinman \& Teachman, 2011) asks participants to read and imagine themselves in two height-relevant scenarios (e.g., climbing a ladder) and then rate the likelihood of eight interpretations related to each scenario (e.g.,

\footnotetext{
${ }^{3}$ The measures reported here are part of a larger study evaluating cognitive bias modification and embodied perception. For example, participants also provided perceptual estimates of heights on a balcony (with measures of peak fear, anxious cognitions, and bodily sensations while standing on the balcony) and completed the phobia section of the SCID a second time in person. For a full listing of measures, please contact the first author.
} 
"You will fall"). Cronbach's alpha was .89 at baseline, and .93 at the post-intervention assessment.

The Recognition Rating Task (modified from Mathews \& Mackintosh, 2000) was used to evaluate whether the interventions influence subsequent novel interpretations. During the post-intervention assessment (but not during the baseline assessment), participants were asked to read and imagine themselves in 10 height-relevant scenarios that were ambiguous with regard to whether the participant's anxiety is manageable or the height is dangerous. Each of the scenarios included a title, and ended with a word fragment to be completed by the participant that was followed by a comprehension question to ensure participants read the scenario. Neither the word fragments nor the comprehension questions resolved the ambiguity of the scenario.

Five minutes after reading the scenarios and completing a distractor task (working on a jigsaw puzzle), participants were shown the title of each scenario, followed by four disambiguated interpretations of each scenario. One interpretation was negative and heightrelevant (labeled "Negative Target") and one was positive and height-relevant (labeled "Positive Target"). The other two were a negative and a positive interpretation that were unrelated to heights or fear (labeled "Foils"). Participants were asked to rate how similar each interpretation was to their recollection of the meaning of the original scenario on a scale of 1 (very different in meaning) to 4 (very similar in meaning). Foils were included to test whether CBM-I specifically altered height-relevant interpretations, or led to a more general positivity bias.

An example of a recognition rating scenario is "THE FOOTBRIDGE: You are walking on a footbridge over a highway. The footbridge is long and you must walk over it for many minutes. As you think about how far you are above the highway, you notice that the footbridge has a low railin_" Disambiguated interpretations for this scenario include: 1) As you traverse the footbridge, you know you are safe despite the fact that the railings are low (Positive Target), 2) As you traverse the footbridge, the low railings make you think that you are unsafe (Negative Target), 3) As you traverse the footbridge, you smile as you feel the warm sun on your face (Positive Foil), and 4) As you traverse the footbridge, you step on a piece of gum (Negative Foil).

\section{Behavioral Approach Task}

The Behavioral Approach Task (BAT) measures fear and approach behavior while the participant climbs a 5-story staircase. Stair-climbing is a typical anxiety provocation in height fear research and has demonstrated treatment sensitivity (e.g., Emmelkamp, Krijn, Hulsbosch, de Vries, Shuemie, \& van der Mast, 2002; Wolitzky \& Telch, 2009). Participants were asked to climb as high on a staircase as they were willing. On each landing, they were asked to complete four tasks: 1) stand near the railing, 2) look at a target placed on the ground below, 3) stand on a short stool $(12.25 \times 10 \times 7.12)$, and 4) look at the target on the ground below while standing on the stool. As done in Steinman and Teachman (2011), approach was measured by adding the number of stories a participant climbed and the percentage of tasks the participant completed on that landing (e.g., a participant who climbed to the third landing and stood near the railing, but refused to look at the target 
below would score a 3.25). Immediately following the BAT, participants were asked to report their peak fear using the PANAS-FS.

\section{Height fear reduction interventions}

In a past exposure-based acrophobia treatment study that allowed for unlimited exposure time, the range of treatment time was 35 to 360 minutes, with a mean of 115 minutes (Bourque \& Ladouceur, 1980). In the current study, participants in all conditions completed a total of 180 minutes of intervention or control tasks, divided between two intervention sessions.

CBM-I-Participants in the CBM-I Only and the CBM-I+Exposure conditions completed modifications of two CBM-I paradigms: Interpretation Modification Program (IMP) and Scenario Training (see Appendix for examples) ${ }^{4}$.

Following Beard and Amir's (2008) IMP paradigm, participants completed 76 trials in which they were trained to associate positive words with ambiguous, height-relevant sentences. Each height-relevant sentence was written to be emotionally ambiguous with regard to one or more of the following: if the participant will be able to handle his/her anxiety, if the participant will be able to accomplish tasks while on a height (e.g., screwing in a light bulb while on a ladder), if the participant finds heights to be dangerous, and if the participant finds heights to be scary. Given that individuals with acrophobia are prone to interpret bodily sensations as threatening (Davey, Menzies, \& Gallardo, 1997), approximately $25 \%$ of the materials in interpretation training tasks referred to ambiguous bodily sensations (e.g., heart racing, sweating) while in a height situation. In each trial, a fixation cross was displayed for $500 \mathrm{~ms}$ in the center of the computer screen. Next, either a positive or negative word appeared in the center of the computer screen for $500 \mathrm{~ms}$, followed by an ambiguous, height-relevant sentence, which remained on the screen until the participant finished reading the sentence and pressed the space bar. For example, following the fixation cross, participants saw either "risky" or "stable." After $500 \mathrm{~ms}$, the word was replaced by "As you stand on a stepladder, you feel it rock slightly beneath you." Next, participants were asked to press " 1 " if they felt the word and the sentence were related, or to press " 3 " if they felt the word and the sentence were not related. Participants received positive feedback ("You are correct") if they responded that positive words were related to the sentences or that negative words were not related to the sentences. Otherwise, they received negative feedback ("You are incorrect."). This contingency was expected to reinforce positive height-relevant interpretations and extinguish negative height-relevant interpretations.

Scenario Training was modeled off of the paradigm developed by Mathews and Mackintosh (2000) but used height-relevant scenarios. In line with prior research (e.g., Steinman \& Teachman, 2010; Teachman \& Addison, 2008), Scenario Training included 64 scenarios that participants were asked to read and imagine themselves in. Each scenario was three sentences long and written to be emotionally ambiguous with regard to the same domains as

\footnotetext{
${ }^{4}$ The valence of all CBM-I and Control training materials, as well as the valence of the disambiguated interpretation options for the Recognition Rating task, were pre-rated by independent samples. Please contact the first author for details on the validation process.
} 
the IMP sentences. At the end of each scenario, participants were asked to complete a word fragment (which had only one solution) that resolved the ambiguity of the preceding scenario in a positive direction. An example of a scenario was, "You are on the roof of a five story apartment building. Grasping the railing, you realize you have never been this high up before. Getting off the roof when you need to will be e_sy." Participants typed the letter "a" to complete the word "easy," ascribing a positive, or non-threatening, interpretation to the preceding text. Following each word fragment, participants answered a comprehension question that reinforced the positive interpretation of the scenario. For the above scenario, the corresponding comprehension question was, "Will it be difficult to safely get off the roof?" Participants typed the letter "n" to indicate "no." Participants were not able to proceed to the next scenario until they correctly completed both the word fragment and the comprehension question.

To ensure that total intervention time was comparable across conditions, the IMP and Scenario Training were repeated during each session as needed. To maintain participants' interest and to enhance learning, subtle variations were made at each repetition (e.g., participants would alternate between reading scenarios aloud or silently, see new comprehension questions in Scenario Training, and see new pairings of words and sentences in IMP).

\section{Control versions of Scenario Training and IMP-Participants in the Control} condition completed a sham variation of Scenario Training and IMP (see Appendix for examples). In this variation, none of the trials were related to height fear. The Control tasks were designed to match the Scenario Training and IMP paradigms for task demands, such as attention, time, format, and other nonspecific factors. To control for effects related to reading about heights and make this condition more credible, approximately $25 \%$ of the trials included height content. However, the height content did not involve fear or emotional ambiguity (in Scenario Training) and was not related to whether the word and sentence were related (in IMP).

Exposure therapy-Participants in the Exposure Only and the CBM-I+Exposure conditions completed an exposure therapy treatment protocol modified from Antony, Craske, and Barlow's (2006) widely used fears and phobia treatment manual. Exposure therapy was comprised of two highly structured sessions, which included: 1) brief psychoeducation about the prevalence, development, and persistence of fears, 2) socialization to treatment, which involved an explanation of habituation and the goals of exposure exercises, and 3) exposure exercises. The exposure exercises took place on various heights throughout the university's campus, such as a balcony, bridge, staircase, and bleachers of a stadium. Participants visited the locations that they were most afraid of based on their personal fear hierarchy. Exposure therapy sessions were led by advanced graduate students that completed extensive training led by the first author (an advanced graduate student) and second author (a licensed clinical psychologist).

The Credibility Scale (modified from Borkovec \& Nau, 1972) is a 3-item measure in which participants' rate how logical the treatment seemed, their confidence that the treatment would eliminate their height fear, and their confidence in recommending the treatment to a 
height fearful friend ${ }^{5}$. Participants in all conditions completed the Credibility Scale after receiving psychoeducation about the rationale for treatment. Participants in the CBMI +Exposure condition completed the Credibility Scale twice: once after the psychoeducation for interpretation training, and once after the psychoeducation for exposure. The average of these two scores was used for this condition. Cronbach's alpha for the 3 items was .77 among participants receiving the Control and Interpretation psychoeducation, and .82 among participants receiving the Exposure psychoeducation.

\section{Procedure}

Prior to study enrollment, all participants completed the AQ-Anxiety and modified SCID questions (either through the psychology department participant pool, or through an online questionnaire). Participants who scored above the established cutoffs were contacted by email to schedule a phone screen. Interested participants were administered the Specific Phobia section of the SCID over the phone, and told that the purpose of the study was to investigate a new, experimental height fear reduction technique, and to compare this new technique to a more traditional fear reduction approach. Participants were assigned to condition with groups balanced for gender ${ }^{6}$. Participants were informed that if they were assigned to the Control condition or a new, experimental condition, they would be given the opportunity to complete the traditional fear reduction approach following conclusion of the study. They were not told which conditions reflected the new versus traditional fear reduction approaches.

Baseline assessment-Following informed consent, participants filled out a brief demographic questionnaire and the PANAS-FS to provide a baseline measure of state fear. Next, participants completed the AQ-Avoidance, ATHQ, and HIQ as baseline measures of height avoidance, attitudes toward heights, and height-relevant interpretation bias. The stairclimbing BAT was then administered, and included completion of the PANAS-FS based on participants' peak fear while doing the BAT.

Height fear reduction/Control intervention sessions-For all participants, the first intervention session took place approximately 24 hours after the baseline assessment. The second intervention session took place approximately 1 week after the first intervention session. Each intervention session was 90 minutes long. In the first intervention session, participants in the CBM-I Only condition were given psychoeducation followed by the Credibility Scale. Next, they completed repetitions of IMP and Scenario Training. The second intervention session repeated the training sequence. For participants in the Exposure

\footnotetext{
${ }^{5}$ We decided to use 3 items, following Taylor and Alden (2011), who also used 3 items from Borkovec and Nau's (1972) treatment expectancy scale.

${ }^{6}$ Participants were sequentially assigned to condition in the following order: Control, Exposure, CBM-I, CBMI+Exposure, and then the sequence would repeat, following a counterbalancing sheet. This was done to make sure that approximately the same number of participants was included in each condition at a constant rate throughout the study (minimizing potential influences of cohort effects), while also insuring that the caseload for the therapists remained relatively stable throughout the study. Note that participants were occasionally assigned to condition out of this order due to scheduling issues (though we did not track which participants were assigned out of this order). However, we find it unlikely that this would have biased the results, especially given that the person handling scheduling was not telling participants which condition they would be assigned to based on their scheduling constraints (e.g., a participant saying she was available on the Tuesday but not the Friday would not know that this might affect her condition assignment because of difficulty booking a therapist for that Friday time). Moreover, the person handling scheduling was not usually the same person who was running the study session, so the experimenter would not know that an assignment had occurred out of order.
} 
Only condition, the first intervention session included psychoeducation, socialization to treatment, (followed by the Credibility Scale) and exposure exercises. The second intervention session consisted of more exposure exercises. For participants in the CBM-I + Exposure condition, the first intervention session included 45 minutes of repetitions of CBM-I (preceded by psychoeducation and the Credibility Scale). Next, participants completed 45 minutes of exposure therapy (which began with psychoeducation and socialization to treatment and the Credibility Scale). CBM-I was offered before exposure therapy because it was expected that participants would practice using the more benign interpretations during the exposure exercises. Moreover, it was predicted that CBM-I would make exposure therapy more palatable. The second intervention session repeated the CBM-I then exposure sequence. For participants in the Control condition, the first intervention session consisted of psychoeducation followed by the Credibility Scale (note that this was the same psychoeducation provided to participants in the CBM-I conditions). Next, they completed repetitions of the Control versions of IMP and Scenario Training to match the training sequence for the CBM-I Only condition. The second intervention session repeated this sequence.

Post-Intervention assessment session-The post-intervention assessment occurred approximately 24 hours after the second intervention session. It was identical to the baseline assessment, except participants also completed the AQ-Anxiety and Recognition Rating Task.

Follow-up-To evaluate durability of the intervention effects, one month after the postintervention assessment, participants received an email asking them to complete an online version of the AQ-Anxiety. After completing the AQ-Anxiety (or approximately two weeks later if they did not respond to the email), participants were debriefed over the phone. Given that Exposure fear reduction procedures are the current gold standard for phobia treatment, following debriefing, an optional 1.5-3 hours of Exposure therapy was offered to all participants not in the Exposure Only condition.

\section{Results}

\section{Descriptive Statistics}

A series of Chi-square tests (for categorical variables) and Analyses of Variance (ANOVAs; for continuous measures) demonstrated that conditions did not differ at the $p=.05$ level for gender, race, age, or pre-intervention symptom and affect measures (AQ-Anxiety, AQAvoidance, HIQ, ATHQ, and peak PANAS-FS on BAT). However, intervention conditions did significantly differ on pre-intervention level of approach during the $\operatorname{BAT}(F(3,104)=$ $\left.3.04, p=.033, \eta_{\mathrm{p}}{ }^{2}=.08\right)$. Specifically, follow-up LSD tests indicated that the CBM-I + Exposure condition showed less approach than the Control condition and the CBM-I Only conditions (both $p \leq .041$ ), and the Exposure condition showed less approach than the CBMI Only condition $(p=.036)$. Additionally, baseline state fear (as measured by the PANASFS) differed between conditions $\left(F(3,105)=3.66, p=.015, \eta_{\mathrm{p}}{ }^{2}=.10\right)$, such that the CBM-I Only condition had lower baseline state fear, relative to the CBM-I+Exposure and Control 
conditions (both $p \leq .047$ ). Note that one extreme outlier was removed from this variable). See Table 1.

In general, very little data were missing in our dataset. Only one participant (in the Exposure Only condition) dropped out of the study following the baseline assessment; this was due to scheduling issues. Including that participants' missing data, only 0-4 (out of a possible 110) participants' data were missing for any of the pre- and post-intervention outcome measures. Further, missingness was not tied to condition. Given that the amount of missing data was small and mostly due to experimenter error, the data were assumed to be missing completely at random. Thus, listwise deletion was used to handle missingness (see Little \& Rubin, 2002). However, given the larger amount of missing data at the one-month follow-up (12 out of 110 participants' data), all missing values for the follow-up AQ-Anxiety were imputed. To impute missing data for the follow-up AQ-Anxiety, the ExpectationMaximization procedure, within the Missing Value Analysis in SPSS Version 21 (IBM Corp. Released 2012), was conducted. This procedure predicted missing values by using the Expectation-Maximization Algorithm and available total scores from the AQ-Anxiety at pre-intervention, post-intervention, and follow-up. Note that Little's MCAR test was not significant $(\chi 2(\mathrm{df}=5)=9.86, p=.079)$, suggesting that Expectation-Maximization was an appropriate imputation procedure.

\section{Credibility of Interventions}

A univariate ANOVA indicated condition differences on the Credibility Scale $(F(3,100)=$ 5.93, $\left.p=.001, \eta_{\mathrm{p}}{ }^{2}=.15\right)$, such that participants in the Exposure condition provided significantly higher credibility ratings compared to the other three conditions $(p \leq .040)$. Also, surprisingly, participants in the Control condition provided higher credibility ratings relative to the CBM-I Only condition $(p=.026)$, despite being provided identical explanations for the interventions. Given these condition differences, we re-ran the primary analyses with credibility ratings as a covariate to check that the Time by Condition interactions did not change. The general pattern of results remained unchanged when credibility was included as a covariate (i.e., the significant interactions outlined below remained significant ${ }^{7}$ ). Consequently, this covariate was not included in the following analyses.

\section{Effects of Intervention Condition}

Primary outcome measures included height fear symptoms and attitudes, interpretation bias, and response to a height stressor. For each outcome measure (except where noted below), a repeated measures ANOVA with one within-subjects factor (Time: Pre-intervention, Postintervention) and one between-subjects factor (Condition: CBM-I Only, Exposure Only, CBM I+Exposure, Control) was conducted. Of note, there was a significant main effect of Time in all analyses, indicating less height fear pathology post-intervention, relative to preintervention (all $p<.01$, unless noted below). For all analyses, Time by Condition interactions were expected, such that the three active conditions would show greater

\footnotetext{
${ }^{7}$ Note that when credibility ratings were included as a covariate, the null Time by Condition interaction for pre-intervention AQ to follow-up AQ became significant $\left(F(3,99)=3.24, p=.025, \mathrm{n}^{2} \mathrm{p}=.09\right)$.
} 
improvement on all outcome measures, compared to the Control condition. For all significant Time by Condition interactions, two follow-up analyses were conducted to determine the source(s) of the interaction: 1) a univariate ANOVA for post-intervention data, with Condition as a between subjects factor, with follow-up LSD tests if a main effect of Condition was revealed, and 2) paired sample t-tests within each Condition, comparing pre-intervention to post-intervention data.

\section{Effects of Intervention Condition on Interpretation Bias}

To evaluate effects on interpretation bias, participants completed the HIQ pre- and postintervention, and Recognition Ratings post-intervention. Results from the repeated measures ANOVA with the HIQ revealed the expected Time by Condition interaction $(F(3,102)=$ $4.78, p=.004, \eta_{\mathrm{p}}{ }^{2}=.12$; see Figure $\left.2 \mathrm{a}\right)$. The follow-up univariate ANOVA suggested condition differences $\left(F(3,102)=6.17, p=.001, \eta_{\mathrm{p}}{ }^{2}=.15\right)$, and follow-up LSD analyses showed that post-intervention, all three active conditions had lower HIQ scores than the Control condition (all $p \leq .005$ ), as expected, and the three active conditions did not significantly differ from one another (all $p \geq .314$ ). Of note, follow-up paired sample $t$-tests revealed that HIQ scores for all four conditions significantly decreased over time (all $p \leq$. 004).

Recognition Ratings were analyzed using a repeated measures ANOVA with two withinsubjects factors (Topic of disambiguated interpretation: Target [i.e., height-relevant], Foil [i.e., non-height-relevant]; and Valence of disambiguated interpretation: Positive, Negative), and the between-subjects Condition factor. We discuss only those effects relevant to Condition, because this was the primary research question. There was a main effect for Condition $\left(F(3,105)=2.85, p=.041, \eta_{\mathrm{p}}{ }^{2}=.08\right)$ and a significant Valence by Condition interaction $\left(F(3,105)=35.77, p<.001, \eta_{\mathrm{p}}{ }^{2}=.51\right)$, which was qualified by the expected three-way Topic by Valence by Condition interaction $\left(F(3,105)=38.64, p<.001, \eta_{\mathrm{p}}{ }^{2}=\right.$. 53). Follow-up ANOVAs indicated significant Condition effects for the two Target interpretations (Negative: $F(3,105)=16.47, p<.001, \eta_{\mathrm{p}}{ }^{2}=.32$; Positive: $F(3,105)=32.89$, $p<.001, \eta_{\mathrm{p}}{ }^{2}=.48$ ), but not the Foil interpretations (both $p \geq .154$ ), pointing to the content specificity of the training effects. Subsequent LSD analyses revealed that both conditions that included CBM (CBM-I Only and CBM-I+Exposure) reported significantly higher endorsements of Positive Target interpretations and lower endorsements of Negative Target interpretations, relative to the two non-CBM conditions (Exposure Only and Control; all $p$ $\leq .001$ ), suggesting CBM altered scenario interpretations as expected. Note that the Exposure Only and Control conditions did not differ from each other, and the CBM-I Only and CBM-I+Exposure conditions did not differ from each other (all $p \geq .093$ ).

\section{Effects of Intervention Condition on Height Fear Symptoms and Attitudes}

Results from the repeated measures ANOVA with height fear as the dependent variable (measured by the AQ-Anxiety) revealed the expected significant Time by Condition interaction $\left(F(3,102)=6.70, p<.001, \eta_{\mathrm{p}}{ }^{2}=.17\right.$; see Figure $\left.2 \mathrm{~b}\right)$. Further, the follow-up univariate ANOVA suggested condition differences $\left(F(3,102)=3.71, p=.014, \eta_{\mathrm{p}}{ }^{2}=.10\right)$, and follow-up LSD analyses indicated that all three active conditions had lower AQ-Anxiety scores than the Control condition (all $\mathrm{p} \leq .009$, except that the difference between Exposure 
and Control did not reach significance; $p=.061$ ). Again, the three active conditions did not significantly differ from one another post-intervention (all $p \geq .263$ ). Additionally, as anticipated, follow-up paired sample $t$-tests revealed that AQ-Anxiety scores for the three active conditions significantly decreased over time (all $p<.001$ ), while the Control condition did not change over time $(p=.121)$.

When evaluating the effects of intervention condition on height avoidance (measured by the AQ-Avoidance), there was a main effect of Condition $\left(F(3,101)=3.75, p=.013, \eta_{\mathrm{p}}{ }^{2}=\right.$. 10 ), such that, on average (collapsed across time points), the Control condition reported more avoidance than the three active conditions (all $p \leq .023$ ), which did not differ from one another (all $p$ 2.559). However, the expected Time by Condition interaction did not reach significance $\left(F(3,101)=1.91, p=.132, \eta_{\mathrm{p}}{ }^{2}=.05\right)$, revealing that there was no differential change in avoidance as measured by the AQ-Avoidance scale across conditions.

The repeated measures ANOVA with attitudes toward heights as the dependent variable (measured by the ATHQ) showed the expected Time by Condition interaction $(F(3,101)=$ $\left.3.13, p=.029, \eta_{\mathrm{p}}{ }^{2}=.09\right)$. The follow-up univariate ANOVA did not suggest condition differences at post-intervention $\left(F(3,102)=2.17, p=.096, \eta_{\mathrm{p}}{ }^{2}=.06\right)$, so follow-up LSD tests were not conducted. However, follow-up paired sample $t$-tests demonstrated that ATHQ scores for the three active conditions significantly decreased over time (all $p \leq .005$ ), while the Control condition did not change over time $(p=.120)$, again as expected.

\section{Effects of Intervention Condition on Response to a Height Stressor}

To determine the effects of intervention condition on responses to a height stressor, participants completed the BAT in the baseline and post-intervention assessments. Results from the repeated measures ANOVA with BAT approach as the dependent variable revealed the expected significant Time by Condition interaction $\left(F(3,103)=4.36, p=.006, \eta_{\mathrm{p}}{ }^{2}=\right.$. 11). The follow-up univariate ANOVA did not suggest condition differences at postintervention $\left(F(3,105)=.61, p=.612, \eta_{\mathrm{p}}{ }^{2}=.02\right)$, so follow-up LSD tests were not conducted. However, as anticipated, follow-up paired sample $t$-tests revealed that participants in the three active conditions showed significantly more approach over time (all $p \leq .009)$, while the Control condition did not change $(p=.218)$.

To evaluate intervention effects on fear in response to a height stressor, standardized residuals were created so that peak fear during the BAT could be calculated while accounting for baseline fear. Specifically, for both baseline and post-intervention assessments, PANAS-FS scores from the beginning of the assessment session were regressed on the PANAS-FS during the BAT scores (see recommendations for examining change in Hummel-Rossi \& Weinberg, 1975). The repeated measures ANOVA with peak fear during the BAT as the dependent variable also showed the expected Time by Condition interaction $\left(F(3,104)=3.17, p=.027, \eta_{\mathrm{p}}{ }^{2}=.08\right)$. Note that this analysis did not show a main effect of time $\left(F(1,104)=.004, p=.953, \eta_{\mathrm{p}}{ }^{2}<.001\right)$. The follow-up univariate ANOVA suggested condition differences $\left(F(3,105)=4.42, p=.006, \eta_{\mathrm{p}}{ }^{2}=.11\right)$, and followup LSD analyses suggested that only the Exposure Only condition reported significantly less fear than each of the other conditions at the post-intervention assessment (all $p \leq .026$ ), while the three other conditions did not differ from one another (all $p \geq .240$ ). Further, 
paired sample $t$-tests revealed that participants in the Exposure Only condition reported significantly less peak fear from baseline to post-intervention $(p=.022)$, while the other three conditions did not change (all $p$ 2.063). Recall that baseline BAT approach and baseline fear differed across conditions, so these analyses should be interpreted with some caution.

\section{Effects of Intervention Condition at a One-Month Follow-Up}

Ninety-eight participants completed the AQ-Anxiety over the Internet approximately one month after the post-intervention assessment. As reported above, missing data for follow-up were imputed. A repeated measures ANOVA with post-intervention and follow-up as the two time points revealed a main effect of Time, such that, on average, participants had lower AQ-Anxiety scores at follow-up than they did at post-intervention $(F(1,102)=13.55, p<$. $\left.001, \eta_{\mathrm{p}}^{2}=.12\right)$. This effect was subsumed by a significant Time by Condition interaction $\left(F(3,102)=4.13, p=.008, \eta_{p}^{2}=.11\right)$. The follow-up univariate ANOVA did not suggest condition differences at the one-month follow-up $\left(F(3,106)=2.18, p=.095, \eta_{\mathrm{p}}^{2}=.06\right)$.

However, follow-up paired sample t-tests revealed that there was no difference between the post-intervention assessment and follow-up for the two conditions including CBM (CBM-I Only and CBM-I+Exposure), suggesting that participants in these conditions maintained their gains (both $p \geq .350$ ). Note that these results should be interpreted with caution, given they rely on interpretation of null results (i.e., no change in scores from post-intervention to follow-up). Results for the other two conditions (Exposure and Control) showed a significant decrease in AQ-Anxiety scores between the post-intervention assessment and follow-up (both $p \leq .002$ ), suggesting further gains for the Exposure group following treatment, and an unexpected, delayed reduction in symptoms for the Control condition. In addition, results of a repeated measures ANOVA with pre-intervention and follow-up as the two time points revealed a main effect of Time, such that, on average, participants had lower AQ-Anxiety scores at follow-up than they did at pre-intervention $(F(1,106)=160.94, p<$. $\left.001, \eta_{\mathrm{p}}^{2}=.60\right)$. However, this analysis did not reveal a significant Time by Condition interaction $\left(F(3,106)=2.38, p=.074, \eta^{2}{ }_{p}=.06\right)$.

Taken together, results suggest that participants in all conditions experienced improvement by the time of the one-month follow-up. However, the improvement occurred earlier for the three active conditions (i.e., at the post-intervention), and the Control condition surprisingly "caught up" by the follow-up assessment.

\section{Reliable Change}

A reliable change criterion of 18.60 was calculated (following Jacobson \& Truax, 1991, and Evans, 1998, using Cronbach's alpha $=.72$ and $\mathrm{SD}=12.73$ for the AQ-Anxiety at preselection). Percentages of individuals displaying reliable change from preselection to the post-intervention assessment for each condition are: $25.93 \%$ for Control, $46.15 \%$ for Exposure, 69.23\% CBM-I Only, and $66.67 \%$ for CBM-I+Exposure. Percentages of individuals displaying reliable change from preselection to follow-up for each condition are: $32.14 \%$ for Control, $67.86 \%$ for Exposure, 59.26\% CBM-I Only, and 62.96\% for CBM-I + Exposure. 
Ombnibus chi-square tests suggested condition differences in the number of participants experiencing reliable change at both the post-intervention $(\chi 2(\mathrm{df}=3)=13.13, p=.004)$ and follow-up $(\chi 2(\mathrm{df}=3)=8.68, p=.034)$ assessments. A series of follow-up chi-square tests were then computed, in which each condition was compared to each other condition (e.g., Control vs. Exposure, Control vs. CBM-I, etc.). For the post-intervention assessment, results suggest that significantly fewer participants in the Control condition experienced reliable change compared to participants in the CBM-I condition $(\chi 2(\mathrm{df}=1)=9.97, p=.002)$ and the CBM-I+Exposure condition $(\chi 2(\mathrm{df}=1)=9.01, p=.003)$, but not the Exposure condition $(\chi 2(\mathrm{df}=1)=2.36, p=.125)$. Of note, the three active conditions did not differ from one another (all $p$ 2.092). At follow-up, significantly fewer participants in the Control condition experienced reliable change compared to participants in the Exposure condition $(\chi 2(\mathrm{df}=1)=7.14, p=.008)$, the CBM-I condition $(\chi 2(\mathrm{df}=1)=4.08, \mathrm{p}=.043)$, and the CBM-I+Exposure condition $(\chi 2(\mathrm{df}=1)=5.24, p=.022)$. Again, the three active conditions did not differ from one another (all $p \geq .508$ )

\section{Mediation}

To determine whether change in interpretation bias mediated the relationship between condition and change in height fear, we used PROCESS, a plug-in for SPSS developed by Preacher and Hayes (2008). Through bootstrapping (1,000 samples), this plug-in determines 95\% confidence intervals for the indirect effect of condition on change in height fear via change in interpretation bias. The mediation effect is determined to be significant $(p<.05)$ if the confidence intervals of the indirect effect do not include zero.

To examine mediation separately for all three active conditions relative to the Control condition, three mediation models were computed. In each model, Condition was the independent variable (CBM-I Only vs Control, Exposure Only vs. Control, and CBMI + Exposure vs. Control), change in AQ-Anxiety between pre- and post-intervention was the dependent variable, and change in HIQ was the proposed mediator. All three models were statistically significant (all $p \leq .002$ ). Additionally, for all three models, the indirect effect of Condition on change in AQ-Anxiety via change in HIQ was significant (i.e., bootstrapping confidence intervals did not include zero, $p<.05$ ). Overall, mediation results suggest that change in interpretation bias is a mediator for each condition's effect on height fear.

\section{Discussion}

The current study evaluated the efficacy of reducing acrophobia symptoms through directly shifting interpretation biases with computerized CBM-I, and tested the clinical utility of CBM-I by comparing and combining it with exposure therapy, the gold standard treatment for specific phobia. All three active conditions (CBM-I Only, Exposure, and CBM-I +Exposure) had mostly similar results. Specifically, all three conditions showed similar reductions in negative interpretation bias as measured by the HIQ, similar reductions in acrophobia symptoms as measured by the AQ-Anxiety, and similar increases in approach as measured by the BAT, and the three active conditions routinely showed greater symptom reduction than the Control condition (as evidenced by either differences at post-intervention, different amounts of change between pre and post-intervention, or both). Notwithstanding, a 
few differences between the active conditions occurred. In this discussion, we review implications of results that were similar across conditions, and then discuss results that diverged from the typical pattern.

As expected, all three active conditions had significantly lower negative interpretation bias as measured by the HIQ, relative to the Control condition. This is in line with past CBM-I studies (see meta-analysis by Hallion \& Ruscio, 2011) and past research demonstrating that cognitive-behavioral treatments, including exposure therapy, lead to change in interpretations (e.g., Teachman, Marker, \& Clerkin, 2010). We find it interesting, but not totally unexpected, that Exposure and CBM-I had similar effects on interpretation bias as measured by the HIQ. This finding highlights how different pathways to fear reduction (e.g., direct modification of bias vs. exposure exercises) can promote similar cognitive change, and is consistent with theory underlying cognitive-behavioral interventions that suggest even so-called behavioral or exposure interventions will reduce cognitive bias (e.g., Beck \& Clark, 1997; Hofmann, 2008). This is the first study to provide evidence that it is possible to shift interpretation bias in a height-fearful sample.

In line with hypotheses, all three active conditions resulted in reductions in both selfreported height fear symptoms as measured by the AQ-Anxiety (although see discussion on null AQ-Avoidance results, below) and attitudes as measured by the ATHQ. Importantly, change in height fear symptoms on the AQ-Anxiety tended to be reliable, based on the evidence of reliable change and maintenance of gains at follow-up. The fact that the CBM-I conditions led to changes in height fear provides support for the causal claim in cognitive models of anxiety (e.g., Beck et al., 1985) that decreasing cognitive bias reduces anxiety. Further, the CBM-I findings suggest that it is possible to reduce fear without activation of negative affect or verbal mediation, two processes previously theorized to be necessary for fear reduction (e.g., Beck \& Clark, 1997; Foa \& Kozak, 1986). Additionally, as hypothesized, all three active conditions displayed an increase in behavioral approach postintervention on the BAT. This provides evidence that both exposure therapy and shifting interpretations can change actual behavior, and not just responses on questionnaires.

Importantly, although all three active conditions showed similar results on most outcome measures, a few differences between conditions occurred. For instance, while all three active conditions showed a similar pattern of change in interpretation bias as measured by the HIQ, a different pattern of results occurred for the Recognition Ratings. Only the conditions including CBM-I (and not the Exposure Only condition) led to significantly more positive and less negative interpretations of novel ambiguous scenarios. A number of differences between the HIQ and the Recognition Ratings may account for the mixed findings. First, there is overlap in the format of the CBM-I Scenario Training and the Recognition Ratings, which may have contributed to the condition differences. Second, it is possible that the Recognition Ratings measure memory bias, rather than interpretation bias, given the delay between reading scenarios and making ratings (see Hertel \& Mathews, 2012). This can be interpreted to suggest that while both interventions successfully manipulate interpretation bias, perhaps only CBM-I paradigms affect memory bias. In fact, Hertel, Vasquez, Benbow, and Hughes (2011) have suggested that modifying memory may be a mechanism underlying CBM-I's effects. 
In line with predictions, at the one-month follow-up, both the CBM-I and CBMI+Exposure conditions maintained their treatment gains, as measured by the AQ-Anxiety, although recall this interpretation is based on a null finding (i.e., no difference between post-intervention and follow-up scores) and should be interpreted cautiously. However, the Exposure Only condition experienced further treatment gains between the post-intervention assessment and follow-up. The reason for the discrepant further gains is unclear. It could be that the groups differed in the extent they engaged in further practice of their new skills (i.e., the Exposure Only group continued to practice tolerating anxiety in height situations, whereas the CBM-I conditions got relatively less practice making non-threatening interpretations once the computerized training program was no longer available). We cannot address this possibility directly with these data, though our findings raise interesting questions about whether further CBM gains could be promoted by more directly advocating ongoing practice of healthy interpretations in new situations after the initial intervention has ended, or by offering booster sessions with the computerized training program. In future studies, assessing the frequency with which individuals visited heights between assessments would help clarify whether group differences in practice affected the results. It is important to note that, despite the condition difference, the CBM conditions did not show decline in treatment gains over time, in line with other findings of durable gains following CBM (e.g., Schmidt, Richey, Buckner, \& Timpano, 2009).

Recall that participants in all conditions experienced similar improvement by the time of the one-month follow-up assessment. However, the improvement occurred earlier for the three active conditions (i.e., at the post-intervention), and the Control condition surprisingly "caught up" by the follow-up assessment. While this could partially reflect some regression to the mean, it is hard to explain why this would occur only at the follow-up and not immediately following treatment. Perhaps a more plausible explanation is that the Control condition involved completion of multiple height exposures as part of the baseline and postintervention assessments, which may have led to some habituation and fear reduction. Nevertheless, the finding that the Control condition showed similar improvement to the active conditions between pre-intervention to follow-up highlights the need for future studies to evaluate ways to strengthen longer-term effects of CBM-I, and suggests that the "maintenance of gains" at follow-up should not be over-interpreted. Further, the generalizability of follow-up data in the current study is limited, given that only one selfreport measure of height fear was used, and no behavioral assessments were conducted.

Finally, while all three active conditions showed increased approach behavior during the BAT at the post-intervention assessment, only the Exposure Only condition differed significantly from the Control condition in level of peak fear during the BAT at the postintervention assessment, as measured by the PANAS-FS. This may be because the BAT was very similar to what was done during the exposure intervention (e.g., many participants practiced standing on stools during exposures). The condition differences in baseline fear may also have played a role, though this variable was accounted for in the peak fear analyses. Notably, the finding that the CBM-I conditions changed their approach behavior but not their peak fear is in line with past research demonstrating uncoupling across multiple systems through which fear can be expressed (Lang, 1978; Lang, Cuthbert, \& Bradley, 
1998), and highlights the need to use multiple outcome measures that tap into various fear systems.

Surprisingly, there was no differential change in self-reported avoidance across conditions on the AQ-Avoidance, despite evidence of differential change in avoidance on the staircase in the BAT, suggesting the two measures assess different facets of avoidance. Of note, results do not suggest self-reported avoidance did not change; rather, they suggest that all conditions (including the Control) experienced reductions in self-reported avoidance. Perhaps individuals in the Control condition experienced reductions in self-reported avoidance due to the exposure to heights that occurred during the assessment sessions.

We did not see the expected synergistic interaction between CBM-I and exposure therapy in the CBM-I+Exposure condition. Although this is contrary to hypotheses, it is in line with past studies suggesting that combining separate efficacious interventions does not enhance treatment effects (e.g., Foa et al., 2005). A growing number of studies have suggested that change in cognition is an active mechanism of change in exposure (e.g., Foa, Franklin, Perry, \& Herbert, 1996; Hofmann, 2004; McManus, Clark, \& Hackmann, 2000). Perhaps both exposure and CBMI affect some of the same mechanisms of fear reduction (i.e., cognitive change); as a result, combining the two interventions is superfluous. This explanation is further supported by our mediation results, which suggest that all three active conditions had an effect on change in height fear via change in interpretation bias. Future studies could counterbalance the order of CBM-I and Exposure, to determine if intervention order affects the hypothesized (but not found) synergistic effect. Perhaps experiencing habituation and realizing that feared consequences do not occur while on a height would make the benign interpretations trained in CBM-I more credible. Finally, understanding the lack of synergy between treatments is complicated by the fact that interventions in the combined condition were each half as long as they were in the individual treatments (i.e., 45 minutes of each intervention vs. 90 minutes of each intervention). It is possible that synergistic effects will only be observed if participants complete longer courses of each intervention.

Despite the few differences seen across outcomes, the overall pattern of results suggests that CBM-I, alone or in combination with exposure therapy, is effective as a treatment for reducing height fear. This is exciting given the potential ease of disseminating the intervention widely, due to the relatively low resources required to administer the intervention (e.g., no therapist time is needed) and its likely cost efficiency. Further, the success of the CBM conditions is especially notable given that this intervention was rated as less credible than exposure prior to treatment. This indicates that CBM's effects do not simply rely on explicit beliefs about its promise, and suggest that demand effects are not likely responsible for the observed pattern of results. It will be important for future research to work on effective ways to enhance the follow-up effects of CBM-I, and evaluate the generalizability of these results to other anxious samples, as well as more diverse samples.

A few caveats should be considered when interpreting these results. First, not all participants in the study had clinical levels of acrophobia. It is possible that results would be different with a diagnosed acrophobic sample; however, this seems unlikely our sample's pre- 
treatment mean on the AQ-Anxiety was very similar (even a little higher) to that of a previous diagnosed acrophobic sample. Second, baseline differences on the BAT compromise interpretation of the behavioral approach outcomes. Third, for the mediation analyses, the study design did not allow for a test of temporal precedence, which is an important component of mediation (see Kazdin \& Nock, 2003). Future studies, involving repeated assessments of both interpretation bias change and symptom change throughout treatment are needed. Fourth, it is possible that the credibility results would have been different if credibility had been assessed after participants had been exposed to the interventions tasks (as opposed to after only hearing about the intervention task rationales/ psychoeducation). Fifth, we used a control condition that is different from many past CBM studies; instead of exposing participants to the same scenarios in both the Control and CBMI conditions (with the contingency established by resolving the word fragments being the only difference between the conditions), we used neutral scenarios for the Control condition. This raises the possibility that the CBM-I in the current study affected height fear through exposure to height-relevant scenarios, rather than through bias modification. However, in a different study (with an obsessional sample), CBM-I-linked reduction in negative interpretation bias was not tied to reductions in physiological responding, or reductions in subjective distress during training (Beadel, Smyth, \& Teachman, in press) suggesting that CBMI does not operate through affective exposure-based mechanisms. Finally, future studies should evaluate durability of CBM-I findings by using more measures at follow-up, such as a BAT (and not just a single questionnaire measure of acrophobia symptoms) and including longer follow-up durations.

Despite these limitations, the current study provides novel evidence that CBM-I can be an effective treatment for height fear, and that it has mostly comparable results to exposure therapy, the current gold standard treatment for phobias.

\title{
Acknowledgments
}

The authors would like to thank Jessica Beadel, Joanna Chango, Meghan Cody, David Fask, Jennifer Green, Erin Miga, and Meg Reuland for their assistance as therapists in this study. The authors would also like to thank Katherine Bian, Celia Cressy, Alexander Cutler, Lauren Faulkner, Victoria Gravett, Barrett Jacobson, Elizabeth Manzella, and Alexandra Werntz for their research assistance. Finally, thanks to Meghan Cody and members of the Teachman Program for Anxiety and Cognition Treatment (PACT) Lab for their helpful feedback and suggestions. This study was supported by NIA R01AG033033 grant awarded to Bethany Teachman, and an Association for Behavioral and Cognitive Therapies (ABCT) Dissertation Award to Shari Steinman.

\section{Appendix: CBM-I and Recognition Rating Examples}

\section{CBM-I Only and CBM-I+Exposure conditions: Scenario Training Examples}

\author{
Scenario
}

You are riding a Ferris wheel at a carnival. When you reach the top, you realize you are so high up that you can no longer see your family down below. This makes you uneasy, but your anxiety can be hand_ed.

\section{Comprehension Question}

Are you able to manage your anxiety? 
Scenario

You are on a flight to Chicago. Looking out of your window, you begin to feel slightly dizzy from looking at the land so far below. You know that having this sensation is nor_al.

Comprehension Question

Is your dizziness a sign of danger?

\section{CBM-I Only and CBM-I+Exposure conditions: Interpretation Modification Paradigm (IMP) Examples \\ Words that may be displayed}

Alarming or Normal

Sentence

You feel short of breath as you are climbing up a fire escape to a $4^{\text {th }}$ story landing.

Words that may be displayed

Alright or Threatening

Sentence

As you are cleaning leaves from your gutter, the ladder you are on makes a creaking sound.

Control Condition: Scenario Training Examples

Non-height-relevant

Scenario-You go to the grocery store. While you are there, you buy eggs, bread, and juice. You forget to purchase $\mathrm{m}_{-} \mathrm{lk}$.

Comprehension Question—Did you remember to buy milk?

Height-relevant

Scenario-You are on a date. You are eating dinner at a restaurant on a rooftop terrace.

You look at your date as the food is served and feel h_ngry.

Comprehension Question-Are you eating at a restaurant?

Control Condition: Interpretation Modification Paradigm (IMP) Examples

Non-height-relevant

Words that may be displayed—Big or Small

Sentence-You watch a tiny ant crawl across the floor. 


\section{Height-relevant}

\section{Words that may be displayed-Cold or Hot}

Sentence-While on a balcony, you feel the warm sun on your face.

\section{References}

Abelson JL, Curtis GC. Cardiac and neuroendicrine responses to exposure therapy in height phobics: Desynchrony within the 'physiological response system.'. Behaviour Research and Therapy. 1989; 27(5):561-567. doi:10.1016/0005-7967(89)90091-0. [PubMed: 2573337]

Antony, MM.; Craske, MG.; Barlow, DH. Mastering your fears and phobias: Workbook. 2nd ed. University Press; Oxford: 2006.

Baker BL, Cohen DC, Saunders JT. Self-directed desensitization for acrophobia. Behaviour Research and Therapy. 1973; 11(1):79-89. doi:10.1016/0005-7967(73)90071-5. [PubMed: 4781961]

Beadel JR, Smyth FL, Teachman BA. Change processes during cognitive bias modification for obsessive compulsive beliefs. Cognitive Therapy and Research. (in press).

Beard C, Amir N. A multi-session interpretation modification program: Changes in interpretation and social anxiety symptoms. Behaviour Research and Therapy. 2008; 46:1135-1141. doi:10.1016/ j.brat.2008.05.012. [PubMed: 18675400]

Beck AT, Clark DA. An information processing model of anxiety: Automatic and strategic processes. Behaviour Research and Therapy. 1997; 35:49-58. doi:10.1016/S0005-7967(96)00069-1. [PubMed: 9009043]

Beck, AT.; Emery, G.; Greenberg, RI. Anxiety disorders and phobias. Basic Books; New York: 1985.

Borkovec TD, Nau SD. Credibility of analogue therapy rationales. Journal of Behavior Therapy and Experimental Psychiatry. 1972; 3(4):257-260. doi:10.1016/0005-7916(72)90045-6.

Bourque P, Ladouceur R. An investigation of various performance-based treatments with acrophobics. Behaviour Research and Therapy. 1980; 18:161-170. doi:10.1016/0005-7967(80)90033-9. [PubMed: 7417204]

Bowler JO, Mackintosh B, Dunn BD, Mathews A, Dalgleish T, Hoppitt L. A comparison of cognitive bias modification for interpretation and computerized cognitive behavior therapy: Effects on anxiety, depression, attentional control, and interpretive bias. Journal of Clinical and Consulting Psychology. 2012; 80(6):1021-1033. doi:10.1037/a0029932.

Choy Y, Fyer AJ, Lipsitz JD. Treatment of specific phobia in adults. Clinical Psychology Review. 2007; 27:266-286. doi:10.1016/j.cpr.2006.10.002. [PubMed: 17112646]

Clerkin EM, Teachman BA. Training interpretation biases among individuals with symptoms of obsessive compulsive disorder. Journal of Behavior Therapy and Experimental Psychiatry. 2011; 42(3):337-343. doi:10.1016/j.jbtep.2011.01.003. [PubMed: 21371415]

Coehlo CM, Santos JA, Silvério J, Silva CF. Virtual reality and acrophobia: One-year follow-up and case study. CyberPsychology and Behavior. 2006; 9(3):336-341. doi:10.1089/cpb.2006.9.336. [PubMed: 16780401]

Cohen, DC. Unpublished doctoral dissertation. Harvard University; Cambridge, MA: 1972. Personality predictors and the outcome of varieties of desensitization.

Cohen DC. Comparison of self-report and behavioral procedures for assessing acrophobia. Behavior Therapy. 1977; 8:17-23. doi:10.1016/S0005-7894(77)80116-0.

Davey GCL, Menzies R, Gallardo B. Height phobia and biases in the interpretation of bodily sensations: Some links between acrophobia and agoraphobia. Behaviour Research and Therapy. 1997; 35(11):997-1001. doi:10.1016/S0005-7967(97)10004-3. [PubMed: 9431729]

Emmelkamp PMG, Bruynzeel M, Drost L, van der Mast CAPG. Virtual reality treatment in acrophobia: A comparison with exposure in vivo. CyberPsychology and Behavior. 2001; 4(3): 335-339. doi:10.1089/109493101300210222. [PubMed: 11710257]

Emmelkamp PMG, Krijn M, Hulsbosch AM, de Vries S, Schuemie MJ, van der Mast CAPG. Virtual reality treatment versus exposure in vivo: A comparative evaluation in acrophobia. Behaviour 
Research and Therapy. 2002; 40(5):509-516. doi:10.1016/S0005-7967(01)00023-7. [PubMed: 12038644]

Evans, C. [Retrieved October 2, 2012] Reliable change criterion calculator. Reliable and clinically significant change. 1998. from http://www.psyctc.org/stats/rcsc1.htm

First, MB.; Spitzer, RL.; Gibbon, M.; Williams, JBW. Structured Clinical Interview for Axis I DSMIV Disorders. Biometric Research Department, New York State Psychiatric Institute; New York: 1994.

Foa EB, Franklin ME, Perry KJ, Herbert JD. Cognitive biases in generalized social phobia. Journal of Abnormal Psychology. 1996; 105(3):433-439. doi:10.1037/0021-843X.105.3.433. [PubMed: 8772013]

Foa EB, Hembree EA, Cahill SP, Rauch SAM, Riggs DS, Feeny NC, Yadin E. Randomized trial of prolonged exposure for posttraumatic stress disorder with and without cognitive restructuring: Outcome at academic and community clinics. Journal of Clinical and Consulting Psychology. 2005; 73(5):953-964. doi:10.1037/0022-006X.73.5.953.

Foa EB, Kozak MJ. Emotional processing of fear: Exposure to corrective information. Psychological Bulletin. 1986; 99(1):20-35. doi:10.1037/0033-2909.99.1.20. [PubMed: 2871574]

Hallion LS, Ruscio AM. A meta-analysis of the effects of cognitive bias modification on anxiety and depression. Psychological Bulletin. 2011; 137(6):940-958. doi:10.1037/a0024355. [PubMed: 21728399]

Hertel PT, Mathews A. Cognitive bias modification: Past perspectives, current findings, and future applications. Perspectives on Psychological Science. 2012; 6(6):521-536. doi: $10.1177 / 1745691611421205$.

Hertel P, Vasquez E, Benbow A, Hughes M. Recollection is impaired by the modification of interpretation bias. Journal of Abnormal Psychology. 2011; 120(4):902-910. doi: 10.1037/ a0023974. [PubMed: 21668083]

Hirsch CR, Hayes S, Mathews A. Looking on the bright side: Accessing benign meanings reduces worry. Journal of Abnormal Psychology. 2009; 118(1):44-54. doi:10.1037/a0013473. [PubMed: 19222313]

Hofmann SG. Cognitive mediation of treatment change in social phobia. Journal of Consulting and Clinical Psychology. 2004; 72(3):392-399. doi:10.1037/0022-006X.72.3.392.

Hofmann SG. Cognitive processes during fear acquisition and extinction in animals and humans: Implications for exposure therapy of anxiety disorders. Clinical Psychology Review. 2008; 28:199-210. doi: 10.1016/j.cpr.2007.04.009. [PubMed: 17532105]

Hummel-Rossi, B.; Weinberg, SL. Practical guidelines in applying current theories to the measurement of change, Parts I and II. Johnson Associates; Greenwich, CT: 1975.

IBM Corp.. Released 2012. IBM SPSS Statistics for Windows. Version 21.0. IBM Corp.; Armonk, NY:

Jacobson NS, Truax P. Clinical significance: A statistical approach to defining meaningful change in psychotherapy research. Journal of Consulting and Clinical Psychology. 1991; 59:12-19. doi: 10.1037/0022-006X.59.1.12. [PubMed: 2002127]

Kazdin AE, Nock MK. Delineating mechanisms of change in child and adolescent therapy: Methodological issues and research recommendations. Journal of Child Psychology and Psychiatry. 2003; 44(8):1116-1129. doi:10.1111/1469-7610.00195. [PubMed: 14626454]

Lang, PJ. Language, imagery, and emotion. In: Pliner, P.; Blankstein, KR.; Spigel, IM., editors. Advances in study of emotion and affect, Vol. 5: Perceptions of emotion in self and others. Plenum; New York and London: 1978. p. 107-117.

Lang PJ, Cuthbert BN, Bradley MM. Measuring emotion in therapy: Imagery, activation, and feeling. Behavior Therapy. 1998; 29(4):655-674. doi:10.1016/S0005-7894(98)80024-5.

Little, RJA.; Rubin, DB. Statistical analysis with missing data. 2nd ed. Wiley; New York: 2002.

MacLeod C, Koster EHW, Fox E. Whither cognitive bias modification research? Commentary on the special section articles. Journal of Abnormal Psychology. 2009; 118(1):89-99. doi:10.1037/ a0014878. [PubMed: 19222317]

Mathews A, Mackintosh B. Induced emotional interpretation bias and anxiety. Journal of Abnormal Psychology. 2000; 109(4):602-615. doi:10.1037/0021-843X.109.4.602. [PubMed: 11195984] 
Mathews A, Ridgeway V, Cook E, Yiend J. Inducing a benign interpretational bias reduces trait anxiety. Journal of Behavior Therapy and Experimental Psychiatry. 2007; 38:225-236. doi: 10.1016/j.jbtep.2006.10.011. [PubMed: 17141179]

McManus F, Clark DM, Hackmann A. Specificity of cognitive biases in social phobia and their role in recovery. Behavioural and Cognitive Psychotherapy. 2000; 28(3):201-209. doi:10.1017/ S1352465800003015.

Menzies RG, Clark JC. Danger expectancies and insight in acrophobia. Behaviour Research and Therapy. 1995; 33:215-221. doi:10.1016/0005-7967(94)P4443-X. [PubMed: 7887882]

Murphy R, Hirsch CR, Mathews A, Smith K, Clark DM. Facilitating a benign interpretation bias in a high socially anxious population. Behaviour Research and Therapy. 2007; 45:1517-1529. doi: 10.1016/j.brat.2007.01.007. [PubMed: 17349970]

Preacher KJ, Hayes AF. Asymptotic and resampling strategies for assessing and comparing indirect effects in multiple mediator models. Behavior Research Methods. 2008; 40:879-891. doi:10.3758/ BRM.40.3.879. [PubMed: 18697684]

Reese HE, McNally RJ, Najmi S, Amir N. Attention training for reducing spider fear in spider-fearful individuals. Journal of Anxiety Disorders. 2010; 24(7):657-662. doi: 10.1016/j.janxdis. 2010.04.006. [PubMed: 20510577]

Schmidt NB, Richey JA, Buckner JD, Timpano KR. Attention training for generalized social anxiety disorder. Journal of Abnormal Psychology. 2009; 118(1):5-14. doi:10.1037/a0013643. [PubMed: 19222309]

Steinman SA, Teachman BA. Modifying interpretations among individuals high in anxiety sensitivity. Journal of Anxiety Disorders. 2010; 24:71-78. doi:10.1016/j.janxdis.2009.08.008. [PubMed: 19747797]

Steinman SA, Teachman BA. Cognitive processing and acrophobia: Validating the Heights Interpretation Questionnaire. Journal of Anxiety Disorders. 2011; 25:896-902. doi:10.1016/ j.janxdis.2011.05.001. [PubMed: 21641766]

Journal of Anxiety Disorders. 26:258-259. Corrigendum published 2012. doi:10.1016/j.janxdis. 2011.09.001.

Teachman BA, Addison LM. Training non-threatening interpretations in spider fear. Cognitive Therapy and Research. 2008; 32(3):448-459. doi:10.1007/s10608-006-9084-z.

Teachman BA, Marker CD, Clerkin EM. Catastrophic misinterpretations as a predictor of symptom change during treatment for panic disorder. Journal of Consulting and Clinical Psychology. 2010; 78(6):964-973. doi:10.1037/a0021067. [PubMed: 20954759]

Watson, D.; Clark, LA. Manual for the positive and negative affect schedule (expanded form). University of Iowa; IA: 1994.

Williams SL, Watson N. Perceived danger and perceived self-efficacy as cognitive determinants of acrophobic behavior. Behavior Therapy. 1985; 16(2):136-146. doi:10.1016/ S0005-7894(85)80041-1.

Wolitzky-Taylor KB, Horowitz JD, Powers MB, Telch MJ. Psychological approaches in the treatment of specific phobias: A meta-analysis. Clinical Psychology Review. 2008; 28(6):1021-1037. doi: 10.1016/j.cpr.2008.02.007. [PubMed: 18410984]

Wolitzky KB, Telch MJ. Augmenting in vivo exposure with fear antagonistic actions: A preliminary test. Behavior Therapy. 2009; 40(1):57-71. doi:10.1016/j.beth.2007.12.006. [PubMed: 19187817] 


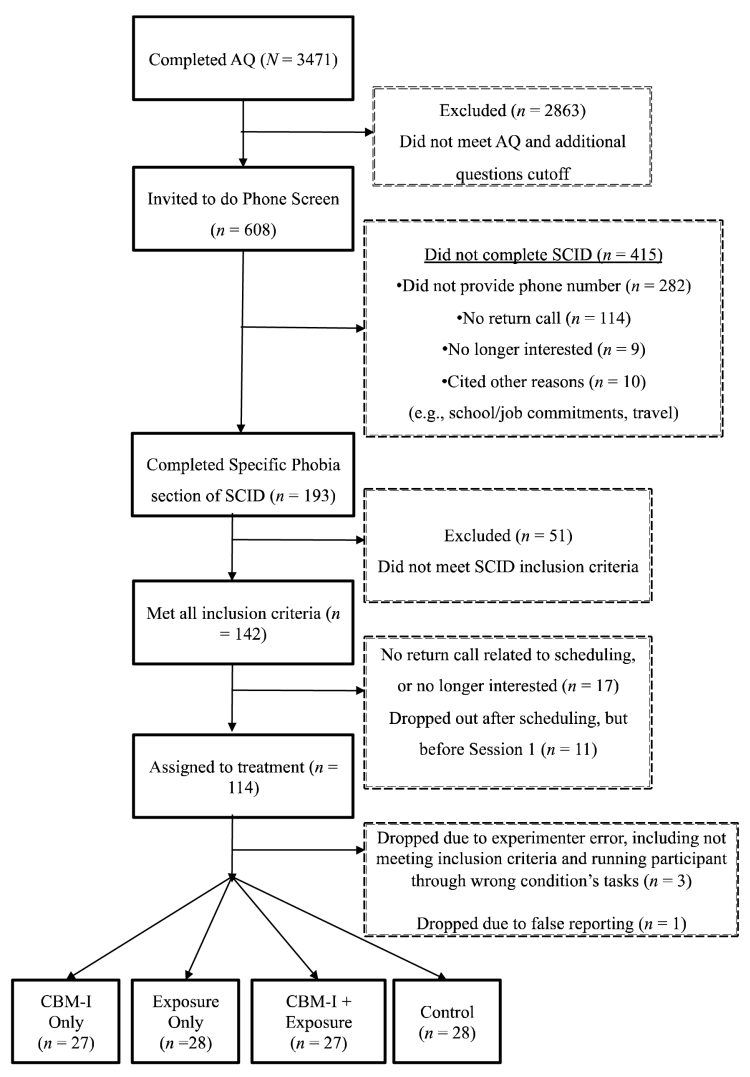

Figure 1.

Consolidated Standards of Reporting Trials (CONSORT) diagram. 


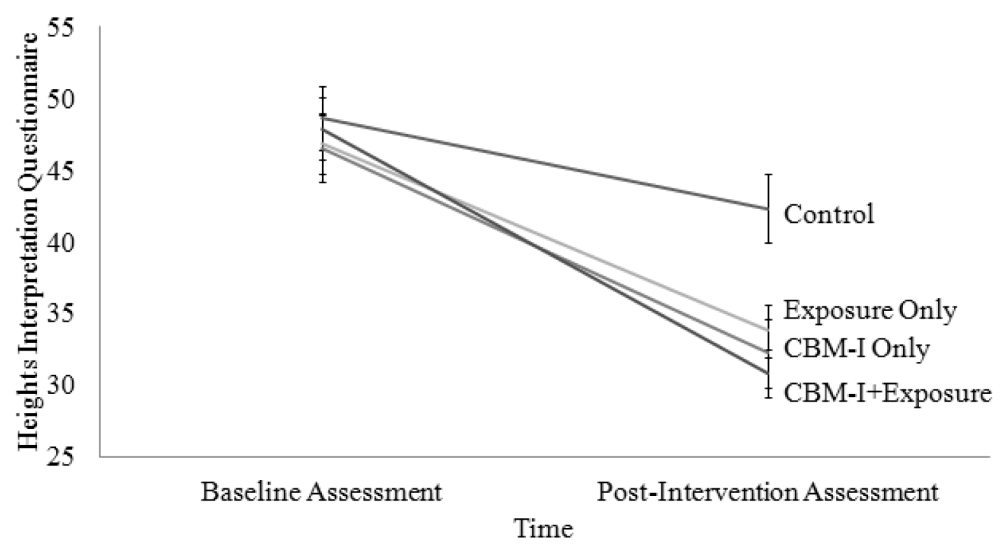

Figure 2a.

Change in Interpretation Bias 


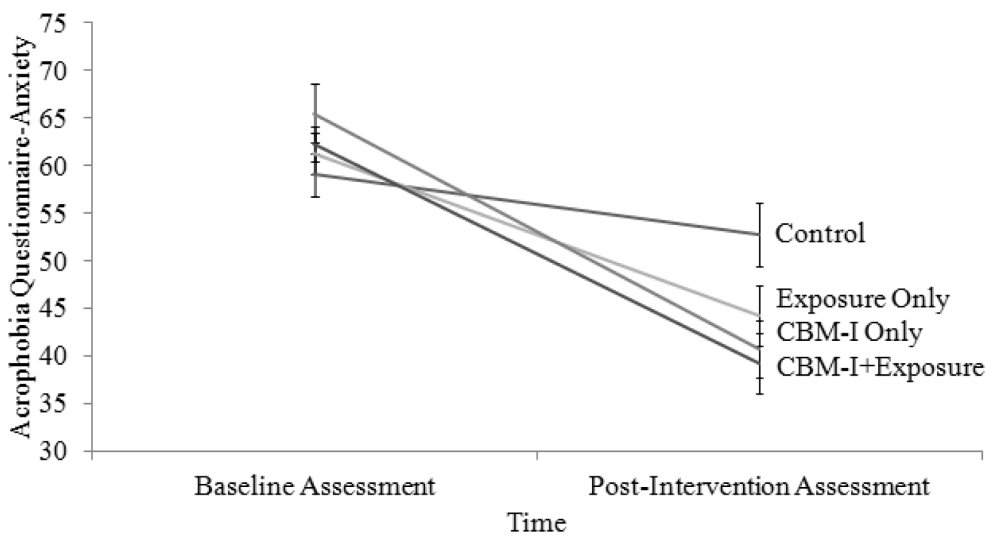

Figure 2b.

Change in Acrophobia Symptoms 


\section{Table 1}

Descriptive Statistics at Baseline.

\begin{tabular}{lcccc}
\hline & $\begin{array}{c}\text { Control } \\
\boldsymbol{M}(\boldsymbol{S D})\end{array}$ & $\begin{array}{c}\text { Exposure } \\
\boldsymbol{M}(\boldsymbol{S D})\end{array}$ & $\begin{array}{c}\text { CBM-I } \\
\boldsymbol{M}(\boldsymbol{S D})\end{array}$ & $\begin{array}{c}\text { CBM-I+Exposure } \\
\boldsymbol{M}(\boldsymbol{S D})\end{array}$ \\
\hline Gender & $75 \%$ Female & $71 \%$ Female & $78 \%$ Female & $78 \%$ Female \\
Age & $21.96(8.19)$ & $27.54(16.66)$ & $24.56(11.79)$ & $20.37(3.74)$ \\
AQ-Anxiety & $60.52(14.18)$ & $61.36(10.68)$ & $65.23(15.55)$ & $62.20(9.85)$ \\
AQ-Avoidance & $17.46(5.00)$ & $15.79(4.40)$ & $15.04(4.31)$ & $15.19(5.26)$ \\
HIQ & $48.71(11.35)$ & $45.97(10.90)$ & $46.04(11.84)$ & $47.81(11.27)$ \\
ATHQ & $38.96(7.75)$ & $39.82(6.48)$ & $39.59(8.92)$ & $38.46(5.50)$ \\
BAT Avoidance ${ }^{*}$ & $4.13(1.00)$ & $3.64(1.13)$ & $4.27(.99)$ & $3.52(1.23)$ \\
$\begin{array}{l}\text { Baseline } \\
\text { PANAS-FS }\end{array}$ & $8.96(2.47)$ & $8.15(1.77)$ & $7.22(1.48)$ & $8.30(1.98)$ \\
$\begin{array}{l}\text { Peak PANAS-FS } \\
\text { on BAT }\end{array}$ & $22.25(4.39)$ & $21.34(5.04)$ & $22.41(6.48)$ & $21.44(5.34)$ \\
\hline
\end{tabular}

Note. AQ-Anxiety = Acrophobia Questionnaire-Anxiety Subscale, AQ-Avoidance $=$ Acrophobia Questionnaire-Avoidance Subscale, HIQ = Heights Interpretation Questionnaire, ATHQ = Attitudes Towards Heights Questionnaire, BAT = Behavioral Approach Task, PANAS-FS = Positive and Negative Affect Schedule-Expanded Form-Fear Subscale.

*significant differences between conditions at baseline. 